\title{
Postural effects on peritoneal transport and systemic uptake of radiolabeled monoclonal antibodies
}

\begin{abstract}
To optimize the regional delivery advantage with i.p. administration of monoclonal antibody $(\mathrm{mAb})$ for radioimmunotherapy, it may be possible to delay the rate and extent of mAb absorption from the peritoneal cavity by simply altering the position of a patient after radioantibody administration. It has been shown that the hydrostatic pressure against the diaphragm plays a major role in the rate of egress of radioantibodies from the peritoneal cavity and that fluid removal from the peritoneal cavity can be altered by posture. The current study examined postural effects in normal rats following the i.p. injection of $125 \mathrm{I}-5 \mathrm{G} 6.4$ murine IgG2a anticarcinoma antibody $(45 \mu \mathrm{Ci})$. A $10-\mathrm{ml}$ injection volume of the radioantibody solution was administered to rats restrained in either a supine or inclined (reverse Trendelenburg; feet down at a $45^{\circ}$ angle) position. Pharmacokinetic analysis confirmed that the appearance of the radioantibody into the systemic circulation was delayed in the inclined group. The time to peak blood concentration was prolonged from 14.7 (supine) to 19.2 (inclined) hours $(P=0.005)$. All other pharmacokinetic parameters were equivalent across the treatment groups. The mean half-life of $166 \mathrm{~h}$, mean blood clearance of $9 \mathrm{ml} / \mathrm{min}$, and mean steady-state volume of distribution of $36 \mathrm{ml}$ were consistent with previous experience with this radioantibody in the rat. The intrinsic absorption profile indicated that the mean percentage absorption from the peritoneal cavity to the blood stream was greater in the supine animals from $4 \mathrm{~h}$ after i. p. injection until absorption was complete. By $10 \mathrm{~h}$ after injection, absorption from the peritoneal cavity was essentially complete in the supine-
\end{abstract}

R. L. W. was supported in part by grants CA33802 and CA42768

J. S. Barrett

DuPont Merck Pharmaceuticals, Department of Drug Metabolism and Pharmacokinetics, Newark, Del., USA

S. J. Fisher - R. L. Wahl (

Division of Nuclear Medicine, Box 0028, Department of Internal Medicine and Department of Radiology, University of Michigan Medical Center, 1500 East Medical Center Drive Ann Arbor, MI 48109-0028, USA dosed animals, while those restrained in an inclined position had cleared only $50 \%$ of the total absorbed dose. Hence, the regional delivery advantage afforded by intraperitoneal administration of the radioantibody may be further exploited by maintaining an inclined position throughout the absorption phase, a strategy that may be applicable to radioimmunotherapy of patients.

Key words Monoclonal antibodies $\cdot$ Pharmacokinetics • Posture · Intraperitoneal

\section{Introduction}

Radiolabeled monoclonal antibodies have been investigated as a treatment modality for advanced, aggressive, and localized tumors not easily eradicated by standard therapy. These agents offer great potential as targeted therapies to normal or pathological sites. They do, however, present unique challenges and opportunities with regard to drug delivery relative to the administration of small drug molecules. Reilly et al. [1] have recently reviewed obstacles and strategies for the delivery of monoclonal antibodies. They cite slow elimination of $\mathrm{mAb}$ from the blood and poor vascular permeability, low and heterogeneous tumor uptake, cross-reactivity with normal tissues, metabolism of $\mathrm{mAb}$ conjugates, and immunogenicity of murine forms in humans as the major problems with mAb delivery. These challenges have limited the efficacy of mAb-based treatment to date, at least for most solid tumors. These not withstanding, regional administration of monoclonal antibodies has reduced some of these concerns.

Intraperitoneal administration of cytotoxic agents targeted to cancers localized in the peritoneal cavity has been explored by many groups recognizing the pharmacokinetic rationale [2] and regional delivery advantage [3, 4] offered by this route of administration. The natural extension of the pharmacokinetic advantage coupled with the allure of the "magic bullet", concept has prompted the production and investigation of radiolabeled mAbs reactive to surface 
antigens on epithelial cancers. While clinical results with i.p. administered radiolabeled $\mathrm{mAb}$ have been mixed [5-7], these studies have validated the regional delivery advantage and reduction in systemic toxicity in the patient population. In addition, investigations into further exploitation of this advantage have provided comparisons of i.p. administered intact antibodies with antibody fragments [8], defined the role of the diaphragmatic lymphatics in peritoneal egress $[9,10]$, and explored the optimization of dosing variables such as injection volume and protein dose [11]. Pharmacokinetic analyses of these studies [8-11] have confirmed that, despite slower rates of absorption in tumor-laden animals, tissue distribution following i.p. administration of murine monoclonal antibodies with preferential reactivity with ovarian and other epithelial cancers as well as nonspecific antibodies was equivalent between normal and tumor-bearing animals, supporting the predictability of exposure data in the normal rat.

It is well known that fluid, particles, and cells are taken up from the peritoneal cavity by lymphatic drainage units located along the peritoneal surface of the muscular portion of the diaphragm in the rat, mouse, cat, dog, and sheep $[12-17]$. On the basis of previous biodistribution studies $[9,12]$, this is the primary route by which radiolabeled monoclonals are cleared from the peritoneal cavity as well. The effect of posture on absorption from the peritoneal cavity has been debated for years. As far back as 1900, Fowler [18] recommended the inclined pelvis-down position for the treatment of peritonitis. Early work by Courtice and Steinbeck [19], suggested that the position of animals after i.p. administration affects the rate and extent of absorption of protein from the peritoneal cavity. Since recent data further suggest that the "pressure head" against the surface of the diaphragm is a major factor influencing diaphragmatic lymphatic uptake, it may be expected that postural conditions may affect the regional delivery advantage afforded i.p. administered radiolabeled mAb. The objective of this study was to examine the effect of posture on the rate and extent of absorption of i.p. administration of a radiolabeled $\mathrm{mAb}$ in the rat, with the expectation that the downward inclined position would slow absorption from the peritoneal cavity and thus enhance the efficacy of regional antibody delivery.

\section{Materials and methods}

\section{Monoclonal antibody}

The intact mouse IgG2aK monoclonal antibody, 5G6.4, was studied. $5 \mathrm{G} 6.4$ is known to react preferentially with ovarian and other epithelial cancers [20]. It was purified using staphylococcal protein A chromatography [21]. The molecular mass and purity of these reagents was verified on $7.5 \%$ sodium dodecyl sulfate/polyacrylamide gels [21].

\section{Radiolabeling}

Labelings were conducted using 125I (New England Nuclear, Boston, Mass.), by the Iodo-Gen method (Pierce Chemical, Rockford, Ill.) [22]. A $1-\mathrm{mCi}$ dose was used to label $100 \mathrm{mg}$ antibody protein. Free iodine was removed from the mixture by anion-exchange resin after labeling. Iodine incorporation was in the $50 \%-80 \%$ range. Freedom from iodine contamination was demonstrated by silica gel thin-layer chromatography with $50 \%$ ethyl acetate and $50 \%$ ethanol [23].

\section{Administration}

Adult female Sprague-Dawley rats (180-225 g) were anesthetized with $50 \mathrm{mg} / \mathrm{kg}$ pentobarbital i.p.; supplemental doses of $15 \mathrm{mg} / \mathrm{kg}$ i. p. were given as required. While pentobarbital can lower the rate of metabolism of small molecules and their portal uptake, this means of anesthesia has not altered peritoneal egress of labeled mAb in previous experiments [9] (and unpublished findings). The rats were then given single i.p. injections of $125 \mathrm{I}$-labeled 5G6.4 in total volume of $10 \mathrm{ml}$ warm $0.01 \mathrm{M}$ phosphate-buffered saline, $\mathrm{pH}$ 7.0. Previous experiments (unpublished) have confirmed that the $10-\mathrm{ml}$ injection volume does not significantly prolong the time to reach maximum systemic concentrations, unlike larger injection volumes $(20 \mathrm{ml})$ in the rat [11]. This volume is not an unreasonable marker for human dosing, as injection volumes of up to 31 have been studied in patients with colon cancer undergoing SPECT scans [24]. Injection-mixture radioactivity levels were measured using a Capintec radioisotope calibrator (CRC-4R). Target doses for the individual administrations were approximately $45 \mu \mathrm{Ci}$ (approximately $1-2 \mu \mathrm{g}$ 5G6.4). Syringe loss accounted for less than $1 \%$ difference from the target administration. Sixteen rats were divided into two groups of eight with the first group remaining lightly restrained in a horizontal (flat, belly-up) position. Rats were weighed and numbered prior to injection. The remaining eight rats were restrained in an inclined (approximately $45^{\circ}$ angle; head-up) position for $24 \mathrm{~h}$. The thyroids of the animals were purposely left unblocked by potassium iodide so that the extent of antibody deiodination could be assessed.

The i.p. injections were administered with a 27-gauge needle attached to a $20-\mathrm{ml}$ syringe. Blood samples were drawn at $0.5,1,2$, $4,6,10,12,14,20,21,22,24,26,48,96,120,144$, and 168 hours after injection. Blood was drawn from the tail vein through pasteur pipettes, placed on glassine paper and weighed using a Mettler balance. Samples were then placed in $12 \times 75-\mathrm{mm}$ test-tubes and capped. At the end of the 7-day sampling period, the rats were sacrificed and their thyroid, liver, kidney, diaphragm, lung, bladder, abdominal wall, and mesenteric, thoracic, femoral, ovarian, and anterior mediastinal lymph nodes were excised. Tissues were rinsed with saline to remove any surface blood and samples of each tissue were weighed on glassine paper. Blood and tissue samples were then counted in a Packard 5780 gamma counter at the $125 \mathrm{I}$ window with the appropriate corrections for count decay. The stability of the labeled antibody in vivo has been previously demonstrated by fast protein liquid radiochromatography [9, 25]. Measured blood counts refer to total 125I activity. Individual blood and tissues levels have been reported in the units of percentage injected dose-kg/g.

Pharmacokinetics

Individual concentration/time profiles from each rat were analyzed via a noncompartmental analysis. Terminal disposition rate constants $(\lambda)$ were estimated by regression of the terminal log/linear concentration/ time points in PC SAS [26]. The terminal disposition half-life was calculated as the quotient of the natural $\log$ of 2 and $\lambda$. The area under the blood concentration/time curve extrapolated to infinity $\left(\mathrm{AUC}_{0-\infty}\right)$ was calculated from the sum of the $\log /$ linear trapezoid, $\mathrm{AUC}_{0-t}$, and the extrapolated trapezoid $\mathrm{AUC}_{t-\infty}$ obtained by dividing the last fitted concentration value from the regression of the terminal phase by $\lambda$. The area under the first-moment curve (AUMC) was similarly calculated. The system mean residence time was calculated as the quotient of the $\mathrm{AUMC}_{0-\infty}$ divided by the $\mathrm{AUC}_{0-\infty}$. Values for the steady-state volume of distribution $\left(V_{\mathrm{d}}\right.$, ss $)$ and systemic clearance $\left(C_{\mathrm{s}}\right)$ were calculated with respect to the bioavailability of the radioantibody. Previous studies with 125I-5G6.4 given i.p. have suggested $100 \%$ bioavailability [8]. The observed parameters, peak concentration $\left(C_{\max }\right)$ and time to peak concentration $\left(t_{\max }\right)$, were also evaluated. 


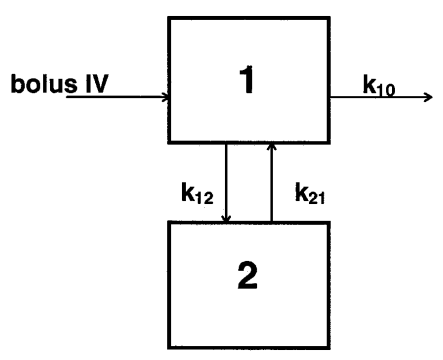

Fig. 1 Two-compartment model scheme with bolus intravenous input and first-order output used to fit plasma profiles from 131I-5G6.4 administered i.v. to Sprague Dawley rats [8]. Micro rate constants: $k_{10}$ is the exit rate constant, $k_{12}$ and $k_{21}$ are distribution rate constants for radioantibody transfer between the blood and peripheral compartments

Descriptive statistics and analysis of variance were conducted on pharmacokinetic-parameter and tissue-distribution estimates using PC SAS for Windows version 6.10 [26]. Tests for homogeneity of variance within the respective treatment groups were also performed.

Data from previous studies [8] in which $20 \mu \mathrm{Ci}{ }^{131}$ I-5G6.4 was administered intravenously to female Sprague Dawley rats was fit to a two-compartment-body model using WinNonlin version 1.0 [27]. The model scheme used to fit the plasma profiles of rats administered ${ }^{131} \mathrm{I}-$ 5G6.4 i.v. is shown in Fig. 1. The estimated parameters $k_{12}$ and $k_{21}$ refer to transfer-rate constants between compartments 1 and 2 and $k_{10}$ is the elimination rate constant. The higher-energy radionuclide, ${ }^{131} \mathrm{I}$, used in the i.v. experiments is not expected to influence the 5G6.4 pharmacokinetics. Mean estimates of the micro-rate constants from the two-compartment model fits, $k_{12}\left(0.049 \mathrm{~h}^{-1}\right), k_{21}\left(0.045 \mathrm{~h}^{-1}\right)$, and $k_{10}$ $\left(0.0045 \mathrm{~h}^{-1}\right)$, determined from the animals undergoing i.v. administration were used with individual concentration/time data following the i. p. administration, to calculate the intrinsic absorption using the LooRiegelman method [28]. The amount absorbed per volume of the central (plasma) compartment was calculated using Eq. 1.

$\frac{A_{T}}{V}=c(t)+k_{10} \int_{0}^{t} c(t) d t+k_{12} e^{-k_{21} t} \int_{0}^{t} c(t) e^{k_{21} t} d t$

The intrinsic absorption, $A_{\mathrm{T}} / V$, was expressed as a percentage by dividing it by the estimated amount absorbed to infinite time, $A_{\infty} / V$, defined by Eq. 2 with conversion to a percentage scale by multiplication by 100 .

$\frac{A_{\infty}}{V}=k_{10} \int_{0}^{\infty} c(t) d t$

Plots of the mean percentage absorption/time profiles were compared across treatment groups by analysis of variance. Pairwise comparisons $(t$-tests) and Fisher's least significant difference across sampling times were also examined.

\section{Results}

Mean ( \pm standard deviation) blood concentration profiles of 125I-5G6.4 in Sprague Dawley rats following i.p. injection in either the inclined or supine position are shown in Fig. 2. Table 1 contains the pharmacokinetic summary of both inclined and supine groups. Both Fig. 2 and Table 1 confirm the equivalence of the treatments with respect to the extent of absorption. Systemic exposure of 125I-5G6.4, as measured by blood AUC, was unaffected by body

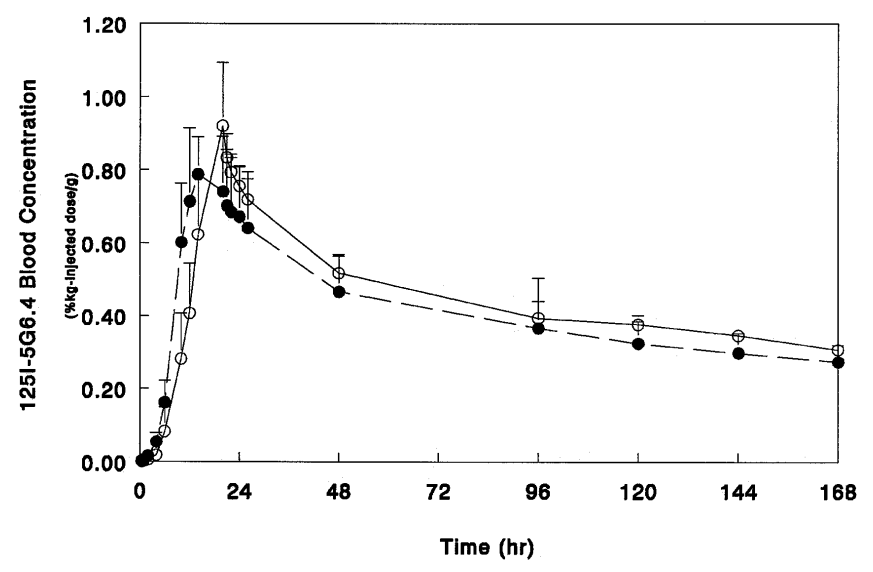

Fig. 2 Mean ( \pm standard deviation) plasma concentrations following single 10-ml i.p. administrations of intact murine IgG2a monoclonal antibody 5G6.4 (approximately $45 \mu \mathrm{Ci}$ ) to female Sprague Dawley rats in supine $(O)$ and inclined $(\bigcirc)$ positions

position. $t_{\max }$, however, was statistically different between the two groups. The grand mean (across groups) half-life of $166 \mathrm{~h}$, grand mean blood clearance of $9 \mathrm{ml} / \mathrm{min}$, and grand mean steady-state volume of distribution of $36 \mathrm{ml}$ were consistent with previous experience with this radioantibody in the rat $[9,11]$. The time course of antibody absorption was altered as Fig. 3 indicates. The intrinsic absorption profile was delayed in the rats maintained in an inclined position after i.p. administration of $125 \mathrm{I}-5 \mathrm{G} 6.4$. The mean percentage absorption was greater in the supine animals from the 4-h sampling time until absorption was essentially complete, as assessed by pairwise comparisons across time. By $10 \mathrm{~h}$ after injection, absorption was completed in the supine-dosed animals, while those restrained in an inclined position had cleared only $50 \%$ of the total absorbed dose. Absorption in the inclined animals was not complete until $14 \mathrm{~h}$ after i.p. injection.

Table 1 Pharmacokinetic summary of single i.p. administration of approximately $45 \mu \mathrm{Ci}$ intact murine IgG2aK monoclonal antibody $125 \mathrm{I}-$ 5 G6.4 to female Sprague Dawley rats in supine and inclined (45 angle) positions. Standard deviations are given in parentheses. $C_{\max }$ maximum concentration in blood and tissue, $t_{\max }$ time to peak concentration, $A U C$ area under the concentration-time curve, MRT mean residence time, $V_{\mathrm{d}}$ ss steady-state volume of distribution, $\mathrm{Cl}_{\mathrm{s}}$ systemic clearance, $F$ bioavailability.

\begin{tabular}{|c|c|c|c|c|}
\hline \multirow[t]{2}{*}{ Parameter } & \multicolumn{4}{|c|}{ Treatment group } \\
\hline & $\begin{array}{l}\text { Supine } \\
(n=5)\end{array}$ & & $\begin{array}{l}\text { Incline } \\
(n=6)\end{array}$ & \\
\hline$c_{\max }(\% \mathrm{~kg}$ injected dose/g) & 0.84 & $(0.15)$ & 0.94 & $(0.16)$ \\
\hline$t_{\max }(\mathrm{h})$ & $14.7 *$ & $(2.5)$ & $19.9 *$ & $(1.7)$ \\
\hline AUC (\% $\mathrm{kg}$ injected dose $\mathrm{h} / \mathrm{g})$ & 131.2 & $(23.3)$ & 148.8 & $(16.6)$ \\
\hline MRT (h) & 231.9 & $(41.9)$ & 249.9 & $(47.9)$ \\
\hline$V_{\mathrm{d}, \mathrm{ss}} / \mathrm{F}(\mathrm{ml})$ & 38.3 & $(7.7)$ & 33.5 & $(2.5)$ \\
\hline $\mathrm{Cl}_{\mathrm{S}} / \mathrm{F}(\mathrm{ml} / \mathrm{min})$ & 10.0 & (1.9) & 8.4 & $(1.2)$ \\
\hline Half-life (h) & 160.3 & $(31.9)$ & 170.4 & $(34.2)$ \\
\hline
\end{tabular}

$* P<0.005$ 


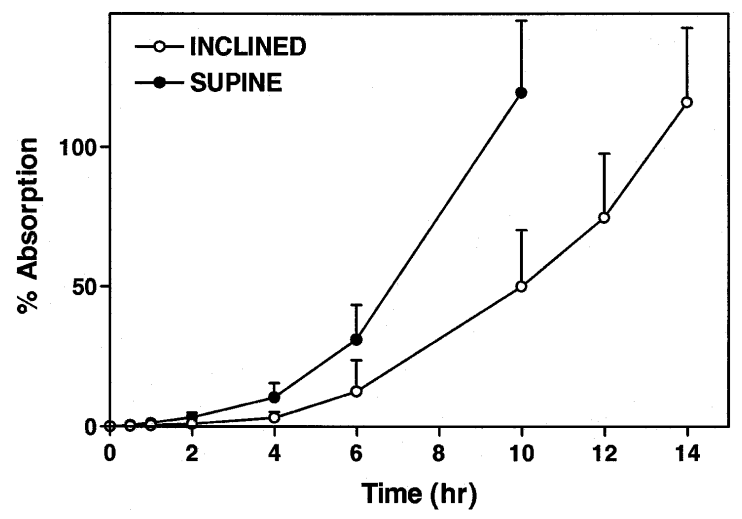

Fig. 3 Mean ( \pm standard deviation) intrinsic absorption (\%) following single 10-ml i.p. administration of intact murine IgG2aK monoclonal antibody 5G6.4 (approximately $45 \mu \mathrm{Ci}$ ) to female Sprague Dawley rats in supine $(O)$ and inclined $(O)$ positions using the Loo-Riegelman method [26]: $k_{10}=0.0046 \mathrm{~h}^{-1}, k_{12}=0.049 \mathrm{~h}^{-1}$, and $k_{21}=0.045 \mathrm{~h}^{-1}$, using data from Wahl et. al. [8] with ${ }^{131} \mathrm{I}-5 \mathrm{G} 6.4$ administered i.v. to Sprague Dawley rats

Interestingly, while the systemic exposure following the single i.p. administrations was equivalent, select tissues excised at sacrifice (7 days after the dose) indicated that the liver, kidney, and mesenteric, thoracic, and anterior mediastinal lymph nodes had significantly $(P<0.05)$ higher tissue uptake in the rats inclined after dosing. Figure $4 \mathrm{~A}$ contains a histogram of mean tissue uptake of 125I-5G6.4, $168 \mathrm{~h}$ after i.p. injection, for both supine and inclined groups. Mean levels of all tissues at sacrifice were slightly higher in the inclined group, likely due to the higher blood levels at late assay times ( $24 \mathrm{~h}$ after initiation of i.p. injection until sacrifice). The significantly higher liver and kidney uptake in the inclined group was thought to reflect the greater deiodination and clearance of free iodine respectively. Figure 4B contains a histogram of the mean thyroid uptake of free ${ }^{125 I}$, $168 \mathrm{~h}$ after i.p. injection, for both the supine and inclined groups, which indicates that, with respect to accumulation of free iodine in the unblocked thyroid, the treatment groups were equivalent. Thyroid levels, however, may not be a sensitive enough marker to detect subtle differences in the deiodination of iodinelabeled $\mathrm{mAb}$ in the liver or the clearance of free iodine via the kidneys, given the difference in magnitude of uptake/organ size between the thyroid and other tissues.

In the inclined group the greater uptake in the mesenteric, thoracic, and anterior mediastinal lymph nodes compared to that in the posterior lymphatics (ovarian and femoral lymph nodes) supports the role of the diaphragmatic lymphatic system in the clearance of i.p. administered $\mathrm{mAb}$ and other proteins from the peritoneal cavity, and further suggests that there is no significant compensation from the posterior mediastinal lymphatics as a result of posture modification. Hence, despite the reduction in pressure head against the diaphragm in the inclined animals, conservation of systemic exposure to the radioantibody was maintained, albeit the intrinsic absorption was delayed and the mechanism of antibody clearance from the peritoneal
A

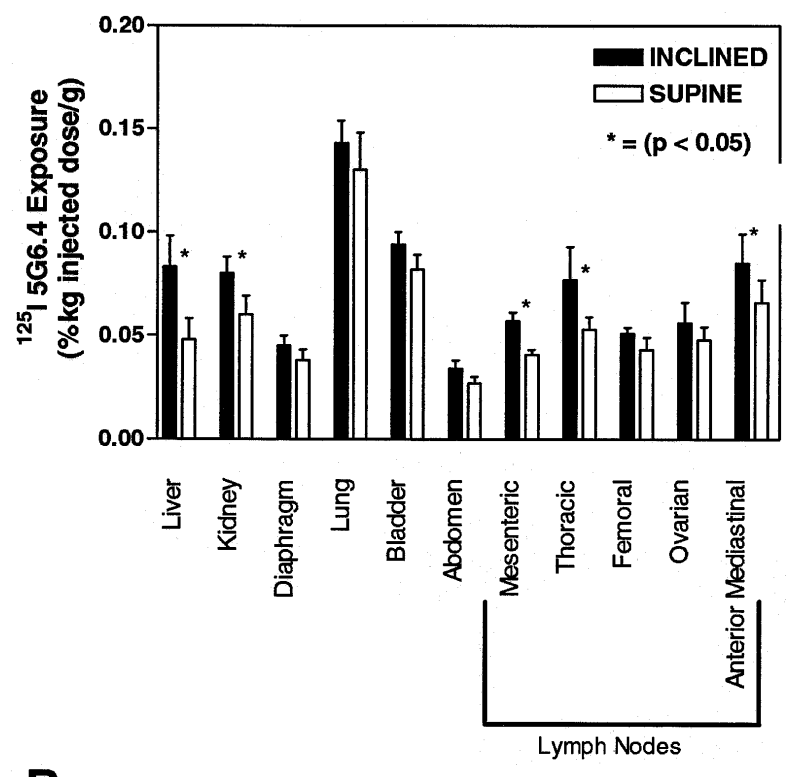

B

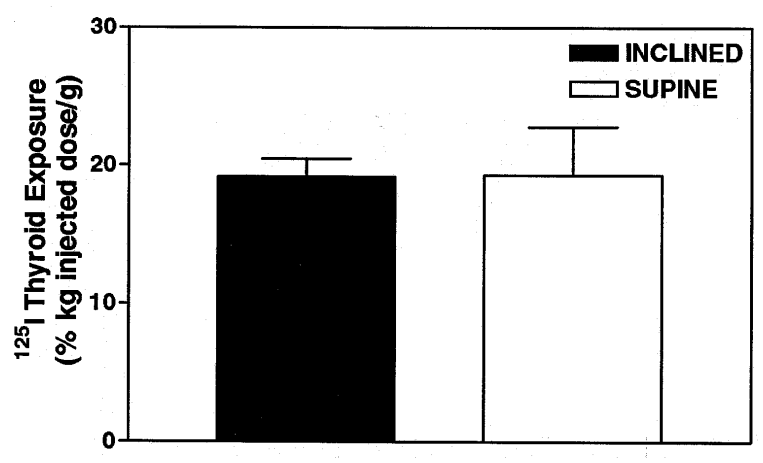

Fig. 4A, B Mean ( \pm standard deviation) (A) tissue uptake of 125I5 G6.4 and (B) thyroid uptake of free ${ }^{125}$ I in female Sprague Dawley rats $168 \mathrm{~h}$ after single 10 -ml i.p. administration (approximately $45 \mu \mathrm{Ci}$ ) in supine or inclined positions

cavity was presumably largely unaffected by posture modifications. Preferential lymphatic uptake after injection likely reflects the longer dwell time of the radioantibody in the peritoneal cavity, but this will require confirmation via serial observations during the absorption phase.

\section{Discussion}

Tumor-reactive monoclonal antibodies labeled with therapeutic radioisotopes offer the potential of targeting radiation therapy with increased cytotoxicity effect and reduced toxicity compared to non-targeted agents. Unfortunately, with the exception of lymphatic tumors, radioimmunotherapy has not been effective in treating most solid tumors following i.v. delivery. Regional administration of these 
agents has broadened the safety-efficacy window. Despite these advances, hematological and gastrointestinal toxicities $[5,6]$ still prevail and the variability in disease burden and injection volume distribution within the peritoneal cavity offer added complications in dosimetry. The relatively poor permeation of radiolabeled $\mathrm{mAb}$ into tumor foci in the peritoneum is a major disadvantage to i.p. treatment.

The present study shows that an inclined position delays the absorption of the radioantibody, $125 \mathrm{I}-5 \mathrm{G} 6.4$, after intraperitoneal administration to the rat. A 4-h difference in the time required for complete absorption was observed between supine and inclined groups. Presumably this behavior is due to the decrease in pressure head (hydrostatic pressure) against the surface of the diaphragm when the i.p. injection volume preferentially rests in the lower portion of the peritoneal cavity. Studies in tumor-bearing animals indicate that the absorption rate of labeled $\mathrm{mAb}$ after i.p. administration is slower relative to that in normal animals [8]. Hence, the effect of posture on peritoneal egress will need to be verified in tumor-involved models and, if favorable, this logically suggests that the regional delivery advantage afforded by i.p. administration can be further enhanced by keeping patients in an upright or inclined position during the time when the injection volume is being absorbed.

Interestingly, the obstruction of subdiaphragmatic lymphatics and the thickening of peritoneal surfaces associated with carcinomatosis can result in protracted clearance of radiolabeled $\mathrm{mAb}$, a phenomenon also observed in the presence of ascites. Notwithstanding the prolonged residence of labeled $\mathrm{mAb}$ in the peritoneal cavity as a result of ascites, the uptake of labeled mAb by tumor nodules has still proved to be small and variable, possibly in part because of free antigen in the ascitic fluid and also because of the short duration of exposure of tumors to high local concentrations of radiolabeled mAb. It may also be that systemic circulation of radionuclide conjugates might increase uptake by vascularized tumor nodules, supporting the notion that increased exposure of the peritoneum may prove relevant for micrometastatic disease.

The use of the inclined posture should potentially enhance the overall uptake of radiolabeled mAb into tumor nodules. Hence, the relevance of this experiment carried out in normal rats may become accentuated in tumor-laden animals and ultimately in patients. Dosing in an inclined position may also provide a greater potential for more complex dosing regimens in the effort to maximize tumor uptake. In summary, the absorption of i.p. administered radioantibodies via longer peritoneal residence times can be delayed in rats following i.p. injection in a reverse Trendelenburg (inclined with feet down at a $45^{\circ}$ angle) versus supine position. This simple physical maneuver may directly enhance the efficacy of intraperitoneal radioimmunotherapy in combination with relatively short-lived tracers or other procedures.

\section{References}

1. Reilly RM, Sandhu J, Alvarez-Diez TM, Gallinger S, Kirsh J, Stern H (1995) Problems of delivery of monoclonal antibodies: pharmaceutical and pharmacokinetic solutions. Clin Pharmacokinet 28: $126-142$

2. Dedrick RL, Myers CE, Bungay PM, DeVita VT (1978) Pharmacokinetic rationale for peritoneal drug administration in the treatment of ovarian cancer. Cancer Treat Rep 62: 1-11

3. Jones RB, Collins JM, Myers CG, et al (1981) High-volume intraperitoneal chemotherapy with methotrexate in patients with cancer. Cancer Res 41: 55-59

4. Speyer JL, Collins JM, Dedrick RL, et al (1980) Phase I and pharmacological studies of 5-fluorouracil administered intraperitoneally. Cancer Res 40: 567-572

5. Muto MG, Finkler NJ, Kassis AI, Howes AE, Anderson LL, Lau CC, Zurawski VR, Weadock K, Tumeh SS, Lavin P, Knapp RC (1992) Intraperitoneal radioimmunotherapy of refractory ovarian carcinoma utilizing iodine-131-labeled monoclonal antibody OC125. Gynecol Oncol 45: 265-272

6. Buckman R, De Angelis C, Shaw P, Covens A, Osborne R, Kerr I, Reed R, Michaels H, Woo M, Reilly R, Law J, Baumal R, Groves E, Marks A (1992) Intraperitoneal therapy of malignant ascites associated with carcinoma of ovary and breast using radioiodinated monoclonal antibody 2G3. Gynecol Oncol 47: 102-109

7. Rubin SC, Kostakoglu L, Divgi C, Federici MG, Finstad CL, Lloyd KO, Larson SM, Hoskins WJ (1993) Biodistribution and intraoperative evaluation of radiolabeled monoclonal antibody MX 35 in patients with epithelial ovarian cancer. Gynecol Oncol 51: $61-66$

8. Wahl RL, Barrett JS, Geatti O, Wilson B, Liebert M, Wagner JG (1988) Intraperitoneal delivery of radiolabeled monoclonal antibodies: studies on the regional delivery advantage. Cancer Immunol Immunother 26: 187-201

9. Barrett JS, Wahl RL, Wagner JG, Brown R, Fisher SJ (1990) Investigations into the route of uptake and pharmacokinetics of intraperitoneally administered monoclonal antibodies I. Transdiaphragmatic blockade of the terminal lymphatics in the rat. Cancer Immunol Immunother 31: 365-372

10. Feldman GB, Knapp RC (1974) Lymphatic drainage of the peritoneal cavity and its significance in ovarian cancer. Am J Obstet Gynecol 119: 991-994

11. Barrett JS, Wahl RL, Wagner JG, Fisher SJ (1991) Investigations into the route of uptake and pharmacokinetics of intraperitoneally administered monoclonal antibodies II. The effect of protein dose and volume of IP injection in the rat. J Cancer Res 51: 3434-3444

12. Olin T, Saldeen $T$ (1964) The lymphatic pathways from the peritoneal cavity: a lymphangiographic study in the rat. Cancer Res 24: 1700-1711

13. Tsilibary EC and Wissig SL (1987) Light and electron microscope observations of the lymphatic drainage units of the peritoneal cavity of rodents. Am J Anat 180: 195-207

14. Courtice FC, Steinbeck AW (1950) The lymphatic drainage of plasma from the peritoneal cavity of the cat. Aust J Exp Biol Med Sci 28: $161-169$

15. Higgins GM, Graham AS (1929) Lymphatic drainage from the peritoneal cavity in the dog. Arch Surg 19: 453-465

16. Feldman GB, Knapp RC, Order SE, Hellman S (1972) The role of lymphatic obstruction in the formation of ascites in a murine ovarian carcinoma. Cancer Res 32: 1663-1666

17. Abernethy NJ, Chin W, Hay JB, Rodela H, Oreopoulos D, Johnston MG (1981) Lymphatic drainage of the peritoneal cavity in the sheep. Am J Physiol 260: F353-F358

18. Fowler GR (1900) Diffuse septic peritonitis, with special reference to a new method of treatment, namely the elevated head and trunk posture, to facilitate drainage into the pelvis, with a report of nine consecutive cases of recovery. Med Rec 57: 617-623

19. Courtice FC, Steinbeck AW (1951) The effects of lymphatic obstruction and of posture on the absorption of protein from the peritoneal cavity. Aust J Exp Biol Med Sci 29: 451-458 
20. Wahl RL, Liebert M, Biesman B, Roberts J, Jackson G, Kronberg S, Laino L (1986) Production and characterization of a murine monoclonal antibody reactive with ovarian and other epithelial carcinomas. Proc Am Assoc Cancer Res 27: 355

21. Laemmli UK (1970) Cleavage of structural proteins during assembly of the head of bacteriophage T4. Nature 227: 680-685

22. Markwell MAK (1982) A new solid-state reagent to iodinate proteins. I. Conditions for the efficient labeling of antiserum. Anal Biochem 125: 427-432

23. Wahl RL, Liebert M, Carey JE, Jackson G (1985) Quality control of radiolabeled monoclonal antibodies: immunological and radiochemical. Cancer Drug Delivery 2: 236
24. Wahl RL, Gyves J, Gross BH, Cochran M, Juni JE, Arnstein NB, Lahti D, Ackermann RJ (1989) SPECT of the peritoneal cavity: method of delineating intraperitoneal fluid distribution. AJR 152: $1205-1210$

25. Himmelsbach M, Wahl RL (1989) Studies on the metabolic fate of ${ }^{111}$ In-labeled antibodies. Int J Radiat Appl Instrum Part B 16: $839-845$

26. SAS Institute, Cary, NC

27. WinNonlin user guide. Version 1.0 (1995) Statistical Consultants Lexington, Ky

28. Loo JCK, Riegelman S (1968) New method for calculating the intrinsic absorption rate of drugs. J Pharm Sci 57: 918-928 